\title{
Recent Regulatory Trends in Pharmaceutical Manufacturing and their Impact on the Industry
}

\author{
Daniel Tabersky, Michael Woelfle, Juan-Antonio Ruess, Simon Brem, and Stephan Brombacher*
}

\begin{abstract}
The pharmaceutical industry is one of the most regulated industries in Switzerland. Though the concept of good manufacturing practises (GMP) was implemented for chemical production in the early 1990s, the rules and regulations for our industry are in constant evolution. In this article we will highlight the impact of these changes to the industry using three recent guideline up-dates as examples: the implementation of $\mathrm{ICH}$ Q3D 'Guideline for elemental impurities', the EU-GMP Guideline Part III Chapter 'Guideline on setting health based exposure limits for use in risk identification in the manufacture of different medicinal products in shared facilities' from 01. June 2015, and the new guidelines to data integrity such as 'PIC/S 041-1 Good Practices for Data Management and Integrity in regulated GMP/GDP environments'. These examples show how scientific approaches help to modernize the control strategies for our products and increase product quality for a better patient safety. The requirements of data integrity regulations are also of interest to industries and universities not working under GXP requirements as they also support the business to improve data quality (traceability) for patent applications, and reduce risk of data falsification.
\end{abstract}

Keywords: ALCOA · Data Integrity · Elemental impurities · Exposure limits · ICH Q3D

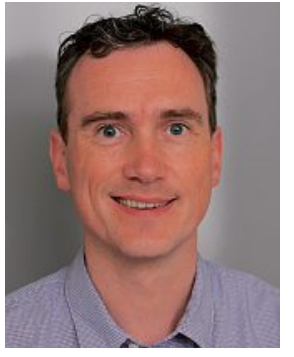

Stephan Brombacher is currently working as Quality Site Head for Novartis Chemical Operations Switzerland. After his $\mathrm{PhD}$ in Environmental Chemistry of the University of Basel, Switzerland he held a PostDoc position at the Marine Bioscience Institute of the NRC in Halifax, Canada, before returning to Switzerland. In Novartis, he held several positions in technical research and development and chemical production in Switzerland.
${ }^{*}$ Correspondence: Dr. S. Brombacher Novartis Campus, WSJ-360.1.02 $\mathrm{CH}-4002$ Basel

E-mail: Stephan.brombacher@novartis.com

\section{Introduction}

The pharmaceutical industry is one of the most regulated manufacturing environments. Since the first implementation of good manufacturing practices (GMP) by the WHO in the 1960 s, to the implementation of PIC/S GMP guide in 1970s, and the later implementation of the EU GMP Guide in 1989 these rules and regulations have been under constant refinement and adaptation. Nowadays, GxP is implemented in all aspects of pharmaceutical manufacturing. This covers technical aspects such as manufacturing of active pharmaceutical ingredients (API) or drug substances, ranging from classic chemical synthesis of small molecules to biomolecules like antibodies, drug product manufacturing such as tablets and solutions for injection, to device manufacturing such as syringes or inhalation devices. In addition, the guidelines also cover aspects such as packaging and distribution of medicines, outsourcing activities, documentation as well as data governance and quality risk management.

InSwitzerland, the 'Arzneimittel-Bewilligungsverordnung, AMBV' from 2001 lists in parallel the PIC/S GMP Guide and the EU GMP Guide as guiding documents for the pharmaceutical industry, both of which are under constant review and revision. In contrast to the opinion that these regulations pose a significant burden to the industry and restrict innovation and flexibility, these rules often induce the de- velopment of new, innovative technologies and more scientific approaches to guarantee the quality and supply security of medicines.

In the following article we would like to highlight this drive to innovation and science by reviewing three recent up-dates on the GMP expectations to industry. We will discuss the requirements and their impact on the pharmaceutical industry with the examples from the production of small molecule active pharmaceutical ingredients (APIs). The first example is the implementation of ICH Q3D 'Guideline for elemental impurities'. Second is the EU-GMP Guideline Part III Chapter 'Guideline on setting health based exposure limits for use in risk identification in the manufacture of different medicinal products in shared facilities' from 01. June 2015, and third are the new guidelines to data integrity such as 'PIC/S 041-1 Good Practices for Data Management and Integrity in regulated GMP/GDP environments'.

\section{Elemental Impurities According to the new Guideline ICH Q3D and USP <232>: A (R)evolution}

The determination of elemental impurities in pharmaceutical products such as residual metals from catalytic reactions or production process-related equipment aberrations is one of the oldest quality control activities performed in the industry. In order to assess the evolution vs. revolution 
of the implementation of ICH Q3D, one has to understand what the historic procedure to determine elemental impurities was and where we are today. For more than 100 years, the elemental impurity profile of a pharmaceutical product was principally assessed by wet chemical and colorimetric limit tests, i.e. according to the formerly valid European Pharmacopoeia (EP) Heavy Metals chapter 2.4.8 ${ }^{[1]}$ and United States Pharmacopeial Convention (USP) General Chapter <231> 'Heavy Metals' . [2] This test utilizes thioacetamide to form coloured complexes of metallic impurities with the sulphide ions with the intensity of the colour of this solution compared against a reference solution. However, these wet chemistry procedures are not element-specific and even highly variable in the response/ sensitivity of the elements and therefore do not deliver the quality and specificity commensurate with the risks attributed to these process impurities. ${ }^{[3-5]}$ The limit for this sum parameter was usually in the order of 10 to $20 \mathrm{mg} / \mathrm{kg}$ and almost all batches of an API were analyzed. This sum parameter can only be used to assess the total heavy metal content semi-quantitatively and only for those elements that were provoked by this test, nevertheless it was established as a routine test for many years. ${ }^{[6]}$

Over the past years, industry consortia, pharmacopoeias, and regulators developed a more effective approach to the control of elemental impurities, leading to a replacement of existing wet chemical and colorimetric tests, EP 2.4.8 and USP <231>. [7] The USP, in parallel with the International Council for Harmonization of Technical Requirements for Pharmaceuticals for Human Use (ICH), has published new standards for measuring and controlling inorganic impurities in pharmaceuticals and their ingredients. The new approach is used for the assessment and control of elemental impurities in the final drug product on the basis of risk management principles as outlined in ICH Q9. ${ }^{[8,9]}$ The ICH method is defined in the 'Guideline for Elemental Impurities' (Q3D), which has been in effect since June 2016 for new marketing authorization applications and was implemented in December 2017 for previously authorized medicinal products. Several Health Authorities have aligned their specific chapters to the content of ICH Q3D, such as the new USP General Chapters $<232>$ (Elemental Impurities - Limits) ${ }^{[10]}$ and <233> (Elemental Impurities Procedures), ${ }^{[11]}$ which were implemented in January 2018. Several papers about measurement procedures were published, see e.g. ref. [4] and references therein. Now we employ state of the art ICP/MS instruments where critical elemental impurities can be controlled down to $50 \mathrm{ppb}$ further reducing the risk for the patient.
In essence, the ICH Q3D consists of three parts: i) the evaluation of toxicity data for potential elemental impurities, ii) the establishment of a Permitted Daily Exposure (i.e. limits) for each element of toxicological concern and iii) application of a risk-based approach to control elemental impurities in drug products (usually done in risk assessments). ${ }^{[8]}$ Thus, the control of elemental impurities in pharmaceuticals transits from a routine testing of concentrations in components to controls based on risk and permitted daily exposures. ${ }^{[7]}$

The evaluation of element- and routespecific toxicological data resulted in permitted daily exposures (PDEs). The PDE is a limit for an elemental impurity in a pharmaceutical product per daily consumption and is dependent on oral, parenteral and inhalational routes of administration. These limits are defined in the ICH and USP chapters and a detailed summary of the deduction of PDEs is given in an appendix to ICH Q3D. ${ }^{[8]}$ Based on their toxicity (PDE) and likelihood of occurrence in the drug product, the elements included in ICH Q3D were divided into three classes. ${ }^{[8]}$ Class 1 consists of the elements $\mathrm{As}, \mathrm{Cd}, \mathrm{Hg}$, and $\mathrm{Pb}$ which are highly toxic to humans and consequently should have limited or no use in the manufacture of pharmaceuticals. Class 2 elements are considered as route-dependent human toxicants and are further divided in two sub-classes (2A and 2B) based on their relative likelihood of occurrence in the drug product. The class 2A elements are: $\mathrm{Co}, \mathrm{Ni}$, and $\mathrm{V}$ and, due to the high likelihood of occurrence, should always be evaluated in a risk assessment. Class 2B elements include: Ag, Au, Ir, Os, $\mathrm{Pd}, \mathrm{Pt}, \mathrm{Rh}, \mathrm{Ru}, \mathrm{Se}$, and $\mathrm{Tl}$ and have reduced risk of occurrence and can only be included if intentionally added to the process. In class 3 are elements with a relatively low toxicity by the oral route of administration (high PDEs, generally $>500 \mu \mathrm{g} /$ day) but it could be necessary to consider those in the risk assessment for inhalation and parenteral routes of administration. Elements that are not included in class 3 are elements with low inherent toxicity. ${ }^{[8]}$

While USP <232> and ICH Q3D apply to all drug products, there is broad industry and regulatory agreement that very few drug products will require routine release testing. However, all products require a documented risk assessment, as defined by ICH Q3D and from this an appropriate control strategy should be deducted that justifies if levels of elemental impurities require additional controls not inherent in the existing control process. The risk assessment should be science-based and connect safety considerations for patients with an understanding of the risk in the product and its manufacturing process. These documents should scientifically justify why elemental impurities testing is not required for the final drug product or define what testing or additional controls need to be added in order to ensure that elemental impurities in the drug product will not exceed the PDE. This justification should specifically address each of the ICH Q3D-defined potential sources of elemental impurities: i) Drug Substance, ii) Excipients, iii) Facilities \& Utilities, iv) Manufacturing Equipment and v) Container Closure System (the packaging used for Drug Products and APIs). ${ }^{[8]}$

In order to ensure compliance with the principles contained in ICH Q3D and USP $<232>$ with respect to control of elemental impurities, Novartis has undertaken a holistic approach focused on an understanding of the factors that may contribute elemental impurities (EI) to the drug product. In developing this comprehensive process, Novartis considered the principal potential sources of elemental impurities described in ICH Q3D. Laboratories across the Novartis network have executed a number of studies to increase the knowledge base and overall understanding of the impact each of these potential sources of elemental impurities may have on the drug product. This resulted in general assessments for manufacturing equipment contributions, low risk excipients, water and container closure systems that can be used as a backbone to all EI product risk assessments. In addition, about 2000 drug product risk assessments were prepared by Novartis employees to ensure compliance with the new regulations.

\section{Implementation of the EMA Guideline on Setting Health-based Exposure Limits}

Another guideline impacting the pharmaceutical industry over the last years was the introduction of health-based exposure limits (HBEL), or permitted daily exposure limits (PDEs), respectively, which had a major impact on cleaning validation processes in the pharmaceutical industry.

In 2014, the European Medicine Agency (EMA) published a guideline on setting health-based exposure limits that was made effective in June 2015. ${ }^{[12]}$ This guideline introduced PDEs as a "substancespecific dose that is unlikely to cause adverse effects if an individual is exposed at or below this dose every day for a lifetime" and is based on toxicological and pharmacological data to ensure safety of human patients. PDEs provide a scientifically justified approach based upon toxicological and pharmacological data to establish carryover limits for active pharmaceutical substances (APIs) in shared pharmaceutical production facilities. Previously, carry- 
over limits were established by applying default limits such as $1 / 1000$ of the minimal daily dose (0.001 MinDD), 10 ppm concentration limit as well as a visual clean criteria. ${ }^{[13,14]}$

While the PDE approach can be considered conservative, there are still objections to the use of PDEs from pharmaceutical industrial groups and some authorities. ${ }^{[14,15]}$ The EMA Q\&A draft document to the guideline demonstrated the difficulties in overcoming traditional limit setting, which resulted in the re-establishment of the 0.001 MinDD limit. ${ }^{[16]}$ For this reason, a pharmaceutical working group was formed in 2017 comprising of toxicology, manufacturing, quality and GMP inspectors to give clear recommendations on limitsetting based upon PDEs. ${ }^{[15]}$ According to the risk management principles in $\mathrm{ICH}$ Q9 "The level of effort, formality and documentation of the quality risk management process should be commensurate with the level of risk". ${ }^{[9]}$ PDEs can be used to identify the highest risk for cross-contamination not only for finished products (APIs) but also for intermediate products and starting material products where dose information is not available but cross-contamination is still a risk.

Also the wider acceptance of healthbased exposure limits has encouraged suppliers of cleaning agents to provide PDEs for their products (e.g. detergents). Due to the inherent low toxicity of the cleaning agents, the carryover limits are less conservative. Limit-setting by previous methods (e.g. $10 \mathrm{ppm}$ concentration limit) did not justify the relatively low hazard properties of these compounds and made their use in cleaning activities difficult.

Introduction of PDEs into API manufacturing of small molecules at Novartis triggered the generation and assessment of a significant amount of toxicological data in order to determine PDEs of all APIs and intermediates. The initial concerns that PDEs could result in considerably lower carryover limits became obsolete upon completion of this toxicological data set. Barle et al. performed a comparison of PDE limits vs. the previous used 0.001 MinDD limit of 140 APIs manufactured by Novartis. ${ }^{[17]}$ The results showed that $91 \%$ of products have a higher maximum allowed carryover using PDEs (Fig. 1). Nevertheless, a small number of products with very low PDEs were observed that mandated an up-date of carryover risk assessments and new cleaning validation activities such as highly active APIs, oncology products or products that possess genotoxic or sensitizing properties that have to be controlled more stringently.

Although the scientific approach of applying PDEs justifies higher carryover limits, this does not mean that equipment

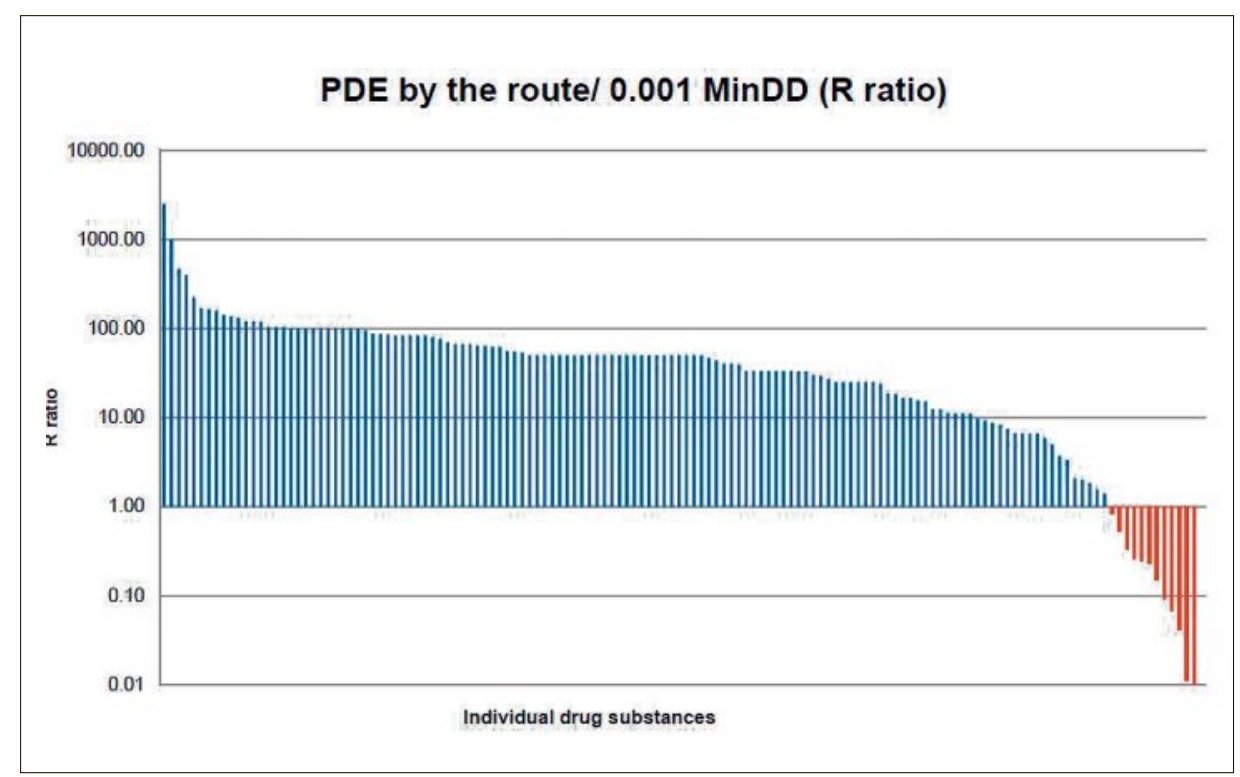

Fig. 1. Comparison of PDE with 0.001 MinDD of the same API. ${ }^{[17]}$

can be left 'dirty' as cleaning limits are only one part of the risk management. The cleaning validation process as specified under GMP guidelines also takes into account criteria such as visually clean, margin of safety, process knowledge, constant improvements and ongoing verification. Even if higher cleaning limits are allowed, meeting the general visual clean requirement may be the most stringent criteria as observable residue levels are usually very low. However the higher limits for cleaning agents and detergents have allowed a wider use of cleaning aids which enables the reduction of solvents for a more environmentally friendly and economic cleaning process in chemical production. ${ }^{[18]}$

\section{Data Integrity Aspects in the Pharmaceutical Industry}

One of the more recent focus areas of health authority inspections is the topic of data integrity. Though the control of data is not a new concept in the GMP regulated environment and already part of several GMP regulations, ${ }^{[18-23]}$ the focus of the documentation aspect of manufacturing processes and analytical work was changed by several publications of health authorities in recent years. In the past there was a strong focus on equipment qualification and the way electronic data is generated and processed in the application as well as where and how it is stored. With the new interpretation of data integrity requirements there is a broader focus on the overall process of data handling and the data lifecycle process from data creation, processing, review, reporting and retention. Thus, the key data integrity question is: "Do I have my data and the data flow under control, from first data generation until the retention of the last data record at the end of my process?"

For example, a fully qualified analytical system can deliver reliable data. However, there might be a high risk of mistakes if the final results are communicated to another production site by phone. Another example, where I also might not be in a position to fully trust the result, would be if the raw data is not protected from manipulation and can be altered without proper traceability.

Therefore, several health authorities or inspecting bodies started to publish dedicated rules and regulations. Three key documents that impacted the industry significantly are, i) the 'MHRA GMP Data Integrity Definitions and Guidance for Industry document in March 2015', ii) the PIC/S Pharmaceutical Inspection Convention/Pharmaceutical Inspection Co-Operation Scheme Draft guidance 'Good Practices For Data Management and Integrity in Regulated GMP/GDP Environments' from August 2016 and iii) the USFDA 'Guidance on Data Integrity'. All these documents guide and mandate the industry to establish well-controlled systems to ensure data integrity throughout the complete manufacturing/analytical process as well as the life cycle of a product from development to fade out of the production.

\subsection{Get a Grasp of the Data Integrity Basics}

One of the key aspects of data integrity is the ALCOA $(+)$ principle. ALCOA is an acronym for: Attributable, Legible, Contemporaneous, Original and Accurate. It has been widely associated with data quality at the FDA and describes the critical attributes to data integrity and docu- 
mentation. The (+) stands for Complete, Consistent, Enduring and Available. These additional terms are based on a European Medicines Agency Guideline Eudralex, Volume 4 , chapter $4^{[22]}$ as well as the aforementioned $\mathrm{PIC} / \mathrm{s}$ draft guidance 'Good Practices for Data Management and Integrity in Regulated GMP/GDP Environments'. In the following we would like to describe the impact of this expectation to an industry from either the lab or manufacturing side (Fig. 2).

- Attributable: Data is expected to be traceable to its primary source and attributable to the individual who observed and recorded it. For electronic data this can be achieved with thorough user access management, audit trail and e-signature.

- Legible: All data recorded must be human-readable and permanent throughout the data lifecycle. This also includes metadata such as audit trail. For example, my handwriting must be readable to others and the way the data is documented must be clearly understandable including added comments, footnotes, margin notes, etc.

- Contemporaneous (at the same time): Information is recorded at the time when the activity is carried out, at the time of data generation and in chronological order. This prevents the risk that an individual is recalling wrong information from memory. Pre-dating or post-dating is not acceptable. Within an electronic system, the audit trail record ensures this by creating timestamps for all data entry and modification.

- Original: The information must be accessible and preserved in its original form as it was created the first time. It is not acceptable e.g. to write an instrument readout on a post-it and transcribe the data to the official reporting document afterwards. The original data is on the post-it note and must be retained as well. Otherwise transcription mistakes might not be detected and retrospective investigation would lack key information.

- Accurate: Data and records should be free from errors, complete, truthful and reflective of the observation. There must be sufficient information to recreate the chain of events without any ambiguity.

These data integrity expectations significantly impact the way data are documented and managed, specifically for processes where data are captured and transferred between paper-based and electronic systems. These so-called hybrid systems usually pose the biggest risk to data transcription, data storage, the ALCOA principles and therefore to the overall data integrity principles.

\section{All forms of GxP data \& records must be ALCOA+}

\section{ALCOA}

\section{Attributable}

Information is captured that identifies the source of the data (audit trails and electronic signatures).

\section{Legible}

Information is human-readable. Reports, tables/listings should be legible.

Contemporaneous (at the same time)

Information is recorded at the time of data generation or event observation. Original

Source of data is available. Data is not a copy (unless $100 \%$ verified) Accurate

Verified as correct via repeatable calculation, algorithm or analysis.

\section{$\mathrm{ALCOA}+$}

\section{Complete}

All data are present, none has been selectively left out.

Available

Anytime, by anyone who needs the data to perform their role

Consistent

Data are compatible, free from variation and non-contradictory.

Enduring

Data are preserved and retrievable during its lifetime according to the date

type retention period.

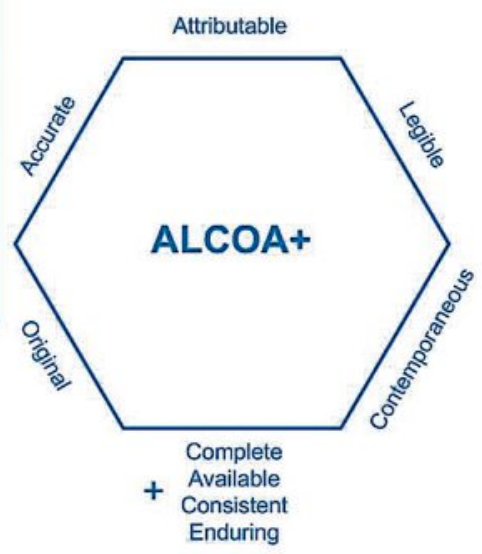

せ NOVARTIS

\subsection{Understanding the Data Processes and the Related Risks}

To understand and manage the data integrity risk of $e . g$. discrepancies between paper and electronic data or undetected data manipulation Novartis implemented a rigorous data process mapping exercise to identify and mitigate data integrity risks. The main objectives of Data Mapping are to systematically describe and understand: - the process, different steps and individual activities,

- the related data flow through systems and equipment, and

- the overall data lifecycle (generation, storage / archival, retrieval, destruction) using a standardized procedure with process- and dataflow diagrams, FMEA risk analysis and resulting action plan. With this approach it was possible to identify weaknesses in manual data recording (people and business process flows) and in data processing and storage in the systems or equipment (data flows). Based on these findings either procedural controls or significant investments into technical solutions, such as new computer systems (and more sophisticated software versions) were put in place to reduce such data integrity risks to an acceptable level or eliminate it completely. The holistic review of all systems in Novartis Chemical Operations Switzerland led to the development of a comprehensive automation strategy to achieve the digital and integrated solution by a step by step implementation as well as harmonization and standardization of processes and techni- cal solutions at the site level. The process automation not only brings significant compliance benefits such as a minimum of manual intervention, improved data insights and a minimized data integrity risk but also enables quality performance improvements of processes and increased efficiency.

\subsection{Foster a Data Integrity-friendly Culture}

Training and data integrity awareness plays a major role in controlling and mitigating data integrity risks. Many data integrity issues observed over the past years were unintentional errors based on missing awareness of the person involved as well as unclear or incomplete guidance documents and procedures. Comprehensive training programs, easy to follow principles as well as feedback session ensure a common understanding of the expectations and lead to a better acceptance and a higher respect of data integrity principles (Fig. 3).

This applies to shop-floor personnel as well as management teams. To ensure data integrity is not just an activity during setup and qualification but is a way of thinking and working with data in our daily life and deeply embedded into the quality DNA of our company.

\section{Conclusion}

New regulations or adaptions to existing guidelines pose a challenge to our daily routines and disrupt our current processes. 


\section{Golden Rules of Data Integrtiy}

$\checkmark$ I lock my computer when I am not present

$\checkmark$ I don't disclose, share or write down my passwords

$\checkmark$ I do not allow another person to operate under my login ID

$\checkmark \quad$ I strictly apply Good Documentation Practices, I don't take any short cuts

$\checkmark \quad$ I record information legibly, clearly and completely

$\checkmark \quad$ I don't use sticky notes or scrap paper to record data

$\checkmark$ I record my activities at the time I perform them

$\checkmark \quad$ I always add the current date to my signature.

$\checkmark$ I never pre-date or back-date signatures.

$\checkmark$ I never delete or throw away GXP data and I report all my data

When I have a question about data integrity, I ask for clarification

$\checkmark$ If I make a mistake, I am open about it and I follow the appropriate procedure

Fig. 3. The 12 golden rules of data integrity.

This requires continuous adaptations to the way we work. On the other hand, the drive for new processes on the basis of thorough scientific principles also fosters innovation and continuously improves the quality of our products to benefit of patients around the world.

\section{Acknowledgements}

I thank my Novartis co-authors for their excellent input that was delivered on top of all the daily work they have to accomplish.

Received: January 27, 2018

[1] European Pharmacopoeia, Edn. 6, Pharm. Eur. 2.4.8, 'Heavy Metals Test', 2008.

[2] United States Pharmacopeia and National Formulary, USP38-NF33, Edn. 38, Chapter $<231>$ 'Heavy Metals', 2015.

[3] N. Lewen, J. Pharmaceut. Biomed. Anal. 2011, 55,653 .

[4] J. S. Barin, P. A. Mello, M. F. Mesko, F. A. Duarte, E. M. M. Flores, Anal. Bioanal. Chem. 2016, 4547 .

[5] N. Lewen, S. Mathew, M. Schenkenberger, T. Raglione, J. Pharmaceut. Biomed. Anal. 2004, $35,739$.

[6] G. H. Fontaine, Chemie Plus 2012, 8, 32

[7] G. Li, D. Schoneker, K. L. Ulman, J. J. Sturm, L. M. Thackery, J. F. Kauffman, J. Pharmaceut. Sci. 2015, 104, 4197.

[8] International Organization for Standardization, 'Q3D Elemental Impurities - Guideline for Industry', Step 4, 2014.

[9] ICH Harmonized Tripartite Guideline: 'Q9: Quality Risk Management', Step 4, 2005.
[10] United States Pharmacopeia and National Formulary, USP39-NF34, Edn. 39, Chapter $<232>$ 'Elemental Impurities - Limits', 2016.

[11] United States Pharmacopeia and National Formulary, USP39-NF34, Edn. 39, Chapter $<233>$ 'Elemental Impurities - Procedures', 2016.

[12] Guideline on setting health based exposure limits for use in risk identification in the manufacture of different medicinal products in shared facilities, European Medicines Agency, 2016, tinyurl.com/y897tls9.

[13] G. Fourman, M. Mullen, Pharmaceut. Technol. 1993, 17(4), 54.

[14] A. Walsh, M. Crevoisier, E. Lovsin Barle, A Flueckiger, D. G. Dolan, M. Ovais, Pharmaceut. Technol. 2016, 40(8), 45.

[15] Questions and answers on implementation of risk-based prevention of cross contamination in production and 'Guideline on setting health based exposure limits for use in risk identification in the manufacture of different medicinal products in shared facilities', European Medicines Agency, 2016, tinyurl.com/y897tls9.

[16] Recognition of HBELs in inspections: Summary of discussions at the workshop on the generation and use of health-based exposure limits (HBEL) held on 20-21 June 2017 at the European Medicines Agency (EMA), EMA/410936/2017.

[17] E. L. Barle, C. Jandard, M. Schwind, G. Tuschl, C. Sehner, D. G. Dolan, Pharmaceut. Technol. 2017, 41, 42.

[18] 'Current good manufacturing practice for manufacturing, processing, packing, or holding of drugs', 21 CFR Part 210, Dec. 2009.

[19] 'Current good manufacturing practices for finished pharmaceuticals', 21 CFR Part 211, Sept. 2014.

[20] 'Electronic Records; Electronic SignatureScope and Application', 21 CFR Part 11, Nov. 2015.

[21] EudraLex, 'The Rules Governing Medicinal Products in the European Union', Vol. 4, 'EU Guidelines for Good Manufacturing Practice for Medicinal Products for Human and Veterinary Use', Jan. 2013, Chap. 1: 'Pharmaceutical Quality System'.

[22] EudraLex, 'The Rules Governing Medicinal Products in the European Union', Vol. 4, 'Good Manufacturing Practice Medicinal Products for Human and Veterinary Use', Jan. 2011, Chap. 4: 'Documentation'.

[23] ICH Harmonized Tripartite Guideline, Jun 2008, Version 4, 'Q10: Pharmaceutical Quality System'. 